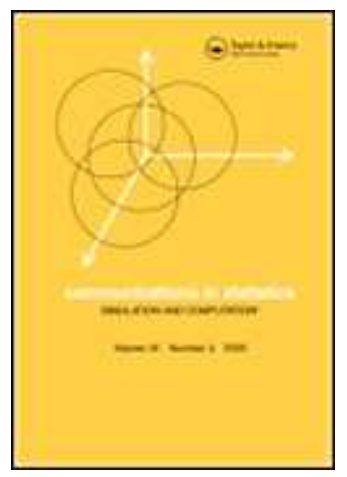

\title{
Maximum likelihood unit rooting test in the presence GARCH: A new test with increased power
}

\begin{tabular}{|c|c|}
\hline Journal: & Communications in Statistics - Simulation and Computation \\
\hline Manuscript ID: & LSSP-2007-0036.R1 \\
\hline Manuscript Type: & Original Paper \\
\hline $\begin{array}{l}\text { Date Submitted by the } \\
\text { Author: }\end{array}$ & $16-$ Oct-2007 \\
\hline Complete List of Authors: & Cook, Steve; Swansea University, Economics \\
\hline Keywords: & Unit roots, GARCH, Monte Carlo, Maximum likelihood \\
\hline Abstract: & $\begin{array}{l}\text { The literature on testing the unit root hypothesis in the presence of } \\
\text { GARCH errors is extended. A new test based upon the combination } \\
\text { of local-to-unity detrending and joint maximum likelihood } \\
\text { estimation of the autoregressive parameter and GARCH process is } \\
\text { presented. The finite sample distribution of the test is derived under } \\
\text { alternative decisions regarding the deterministic terms employed. } \\
\text { Using Monte Carlo simulation, the newly proposed ML t-test is } \\
\text { shown to exhibit increased power of relative to rival tests. Finally, } \\
\text { the empirical relevance of the simulation results is illustrated via an } \\
\text { application to real GDP for the UK }\end{array}$ \\
\hline \multicolumn{2}{|c|}{$\begin{array}{l}\text { Note: The following files were submitted by the author for peer review, but cannot be converted } \\
\text { to PDF. You must view these files (e.g. movies) online. }\end{array}$} \\
\hline GLS_GARCH_2.tex & \\
\hline
\end{tabular}

\section{S) ScholaroNE \\ Manuscript Central}




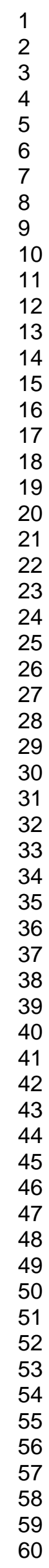




\title{
Maximum likelihood unit rooting test in the presence GARCH: A new test with increased power
}

\author{
Steven Cook* \\ October 16, 2007
}

\begin{abstract}
The literature on testing the unit root hypothesis in the presence of GARCH errors is extended. A new test based upon the combination of local-to-unity detrending and joint maximum likelihood estimation of the autoregressive parameter and GARCH process is presented. The finite sample distribution of the test is derived under alternative decisions regarding the deterministic terms employed. Using Monte Carlo simulation, the newly proposed ML $t$-test is shown to exhibit increased power of relative to rival tests. Finally, the empirical relevance of the simulation results is illustrated via an application to real GDP for the UK.
\end{abstract}

Keywords: Unit roots; GARCH; Monte Carlo simulation; Maximum likelihood estimation; GDP. JEL codes: C12; C15.

\footnotetext{
* Professor Steven Cook, School of Business and Economics, Swansea University, Singleton Park, Swansea, SA2 8PP. Tel: (01792) 602106. E-mail: s.cook@swan.ac.uk.
} 


\section{Introduction}

It has long been recognised that financial time series exhibit volatility clustering. In response, Engle (1982) introduced the notion of autoregressive conditional heteroskedasticity (ARCH) as a means capturing this phenomenon via modelling of the temporal dependency of the conditional variances of time series processes. This research was subsequently extended in the independent studies of Bollerslev (1986) and Taylor (1986) where the generalised autoregressive conditional heteroskedasticity $(\mathrm{GARCH})$ model was proposed. The GARCH model and its various modifications now occupy as central position in empirical and theoretical research, exhibiting attractive properties in widespread application to financial data (see Anderson and Bollerslev 1998). In recent years the noted prevalence of GARCH in financial time series has led to the emergence of a literature examining its impact upon examination of the unit root hypothesis, which following the seminal work of Dickey and Fuller (1979) (DF) has itself become a cornerstone of empirical research in time series econometrics. In its simplest case, the DF test examines the unit root hypothesis $\left(\mathrm{H}_{0}: \rho=1\right)$ in the equation below:

$$
y_{t}=\rho y_{t-1}+\varepsilon_{t}
$$

where $y_{t}$ denotes a variable of interest and $\varepsilon_{t}$ is an error term. Following DF, an enormous literature has emerged examining and extending the analysis of the unit root hypothesis in a variety of ways. The various developments made include examination of unit root testing in the presence of structural change (see, inter alia, Perron 1989, 1997; Leybourne et al. 1998; Leybourne and Newbold 2000; Cook 2002) and the use of alternative estimators to increase test power and/or reduce bias in the estimation of the autoregressive parameter $\rho$ (see, inter alia, Pantula et al. 1995; Park and Fuller 1995; Shin and So 2001). For a full discussion of the literature on the testing of the unit root hypothesis, the reader is referred to the excellent survey provided by Maddala and Kim (1999).

Research involving the joint consideration of the unit root hypothesis and GARCH behaviour has followed two approaches. Under the first approach, investigators have examined the behaviour of a range of unit root tests when GARCH is present but not addressed by the practitioner. Examples 
of studies considering the impact of neglected GARCH behaviour upon unit root tests include Kim and Schmidt (1993), Haldrup (1994), Brooks and Rew (2002) and Cook (2006a, 2006b, 2006c). The general conclusion to be drawn from these studies is that while GARCH has little impact upon the size of linear unit root tests such as those of Dickey and Fuller (1979), Elliott et al. (1996), Granger and Hallman (1991), Leybourne (1995), Park and Fuller (1995), Perron (1997) and Shin and So (2001), asymmetric and non-linear unit root tests (see Enders and Granger 1998, Enders 2001, Cook and Manning 2003 and Kapetanios et al. 2003) can suffer severe size distortion in the presence of GARCH. In contrast to this work, the second approach to the analysis of the interaction between unit root testing and GARCH adopts a different stance whereby the presence of GARCH is explicitly addressed by the practitioner. This research is exemplified by Seo (1999) where it is suggested that in the presence of GARCH, a higher powered approach to testing of the unit root hypothesis is possible via joint maximum likelihood (ML) estimation of the autoregressive (AR) unit root and a GARCH process. The results of this analysis show that under ML estimation of a DF testing equation and a GARCH process, the asymptotic distribution of the $t$-statistic for the unit root hypothesis is a mixture of the non-standard DF distribution and the standard normal. The weightings attached to each of these distributions is dependent upon the strength of the GARCH effect which is captured by a nuisance parameter $(\rho)$. As the GARCH effect increases and $\rho$ moves from its lower limit of 0 towards its upper limit of 1 , the asymptotic distribution of the ML $t$-statistic moves from the DF distribution towards the standard normal. In subsequent research, Cook (2007) has examined the relevance of these asymptotic arguments for the types of finite sample encountered in empirical research. Using Monte Carlo simulation, it was shown that the proposed movement of the distribution of the resulting ML $t$-statistic from the DF distribution towards the standard normal is dependent upon both a very large sample size and an empirically large value for the volatility parameter of the GARCH process. In response to this, the finite-sample distribution of the ML $t$-statistic under joint estimation of a DF testing equation and GARCH process, denoted as DF-GARCH, was derived for alternative sample sizes and values of the GARCH parameters. The resulting critical values of the DF-GARCH test were found to vary substantially according to both the sample size and value of the volatility parameter of the GARCH process. 
In this paper the research of Seo (1999) and Cook (2007) is extended via the proposal of a new unit root testing procedure which introduces local-to-unity detrending to the joint estimation of a unit root testing equation and GARCH process. Following Elliott et al. (1996) it is recognised that local-to-unity detrending via generalised least squares (GLS) results in an increase in power of the DF unit root test. The purpose of this paper is derive a ML $t$-statistic which, under GLS local-tounity detrending, jointly estimates the AR parameter and a GARCH process. The distribution of this newly proposed DF(GLS)-GARCH statistic is derived and its power properties compared to those of the DF and DF-GARCH tests. It is shown that the newly proposed test has a clear power advantage over its rivals.

This paper proceeds as follows. Section [2] presents the newly proposed DF(GLS)-GARCH statistic. In section [3] Monte Carlo simulation is employed to derive the finite-sample distribution of the test under alternative decisions concerning the inclusion of deterministic terms. The empirical power of the DF(GLS)-GARCH test is examined via comparison with the DF and DF-GARCH tests in section [4]. The simulation power results receive an empirical illustration in section [5] via an analysis of the order of integration of UK real output. Section [6] concludes.

\section{GLS-based unit root testing incorporating GARCH}

Examination of the unit root hypothesis in the presence of GARCH, as conducted by Seo (1999) and Cook (2007) can be illustrated by the following system of equations:

$$
\begin{aligned}
\Delta y_{t} & =d_{t}+\beta y_{t-1}+\varepsilon_{t} \quad t=1, \ldots, T \\
h_{t}^{2} & =\phi_{0}+\phi_{1} \varepsilon_{t-1}^{2}+\phi_{2} h_{t-1}^{2} \\
\varepsilon_{t} & =h_{t} v_{t} \\
v_{t} & \sim \mathrm{N}(0,1)
\end{aligned}
$$

where $d_{t}$ is a deterministic component which is specified as either an intercept $\left(d_{t}=\alpha_{0}\right)$ or an intercept and linear trend term $\left(d_{t}=\alpha_{0}+\alpha_{1} t\right)$ and $\varepsilon_{t}$ is a $\operatorname{GARCH}(1,1)$ error process. This representation draws upon the Monte Carlo simulation design of Seo (1999) and Cook (2007). ${ }^{1}$ Following ML estimation of the above model, the unit root hypothesis $\left(H_{0}: \beta=0\right)$ is tested via the $t$-ratio

\footnotetext{
${ }^{1}$ Note that while Cook (2007) explicitly includes deterministic terms in the testing equation of (2), Seo (1999) instead considers initial demeaning or detrending of the series of interest via preliminary regression.
} 
of $\beta$ in (2), the resulting test being denoted here as $t_{\beta}$. Simulation results presented by Seo (1999) show the $t_{\beta}$ test to exhibit a gain in power relative to estimation of the DF testing equation of (2) alone. To complement the primarily asymptotic analysis of Seo (1999), Cook (2007) shows the finite-sample distribution of $t_{\beta}$ to be a function of the sample size $(T)$ employed and values of the GARCH parameters $\left\{\phi_{1}, \phi_{2}\right\}$. In particular it is shown that the convergence of the distribution of the $t_{\beta}$ statistic towards the standard normal is dependent upon large values of $\phi_{1}$ and $T$.

In this paper the above framework is modified via the introduction of local-to-unity detrending using GLS in an attempt to increase the power of the resulting ML $t$-statistic. The modification therefore relates to the treatment of the deterministic term $\left(d_{t}\right)$ in $(2)$ above, thus representing a direct extension of the work of Elliott et al. (1996) for the DF test. Adopting the approach of Elliott et al. (1996), a deterministic process $z_{t}$ is introduced which is specified as $z_{t}=1$ if the series of interest $\left(y_{t}\right)$ is to be demeaned, or $z_{t}=(1, t)^{\prime}$ if detrending is appropriate. With the relevant deterministic term $z_{t}$ decided upon, quasi-differenced data are generated as:

$$
\begin{aligned}
& y_{\bar{\alpha}}=\left[y_{1}, y_{2}-\bar{\alpha} y_{1}, \ldots, y_{T}-\bar{\alpha} y_{T-1}\right]^{\prime} \\
& z_{\bar{\alpha}}=\left[z_{1}, z_{2}-\bar{\alpha} z_{1}, \ldots, z_{T}-\bar{\alpha} z_{T-1}\right]^{\prime}
\end{aligned}
$$

where $\bar{\alpha}=1+\bar{c} T^{-1}$ and $\bar{c}$ is a constant determining the extent of local-to-unity detrending. The GLS detrended series $\widetilde{y}_{t}$ is then derived as $\widetilde{y}_{t}=y_{t}-\widehat{\beta}_{0}$ when $z_{t}=1$, and $\widetilde{y}_{t}=y_{t}-\widehat{\beta}_{0}-\widehat{\beta}_{1} t$ when $z_{t}=(1, t)^{\prime}$, with the $\widehat{\beta}_{i}$ coefficients obtained from the regression of $y_{\bar{\alpha}}$ upon $z_{\bar{\alpha}}$. The extent of quasi-differencing is therefore dependent upon the value of $\bar{c}$. Following Elliott et al. (1996) $\bar{c}=-7$ is imposed for the intercept model, while $\bar{c}=-13.5$ for the trend model. ${ }^{2}$ The unit root hypothesis is then tested via the ML $t$-ratio for $\gamma$ in (8), with the framework of (2)-(5) revised as (8)-(11) below:

$$
\begin{aligned}
\Delta \widetilde{y}_{t} & =\gamma \widetilde{y}_{t-1}+\varepsilon_{t} \quad t=1, \ldots, T \\
h_{t}^{2} & =\phi_{0}+\phi_{1} \varepsilon_{t-1}^{2}+\phi_{2} h_{t-1}^{2} \\
\varepsilon_{t} & =h_{t} v_{t} \\
v_{t} & \sim \mathrm{N}(0,1)
\end{aligned}
$$

\footnotetext{
${ }^{2}$ The stated values of the quasi-differencing parameters are employed also by Elliott et al. (1996) when extending the Dickey-Fuller test and Rodriguez and Perron (2001) when extending the Engle-Granger test.
} 
The resulting test statistic is then denoted as $t_{\gamma}^{G L S}$. Application of this test therefore involves an initial exmination of the properties of the series under investigation to ascertain whether the test should be employed in its intercept $\left(z_{t}=1\right)$ or trend $\left(z_{t}=(1, t)^{\prime}\right)$ forms. With this decided, the appropriate GLS detrending is undertaken using (6)-(7), before the unit root hypothesis is tested using (8)-(11).

\section{$3 \quad$ Finite-sample distribution of the $t_{\gamma}^{G L S}$ statistic}

To examine the finite-sample distribution of the $t_{\gamma}^{G L S}$ statistic, the following data generation process (DGP) is employed:

$$
\begin{aligned}
y_{t} & =y_{t-1}+w_{t} \quad t=1, \ldots, T \\
h_{t}^{2} & =\phi_{0}+\phi_{1} w_{t-1}^{2}+\phi_{2} h_{t-1}^{2} \\
w_{t} & =h_{t} v_{t} \\
v_{t} & \sim \mathrm{N}(0,1)
\end{aligned}
$$

In previous research, Cook (2007) showed the finite-sample distribution of the DF-GARCH test to be highly dependent upon the values of the parameters of the GARCH process $\left(\phi_{1}, \phi_{2}\right)$. In particular, it was found that the distribution was driven by the value of the volatility parameter $\phi_{1}$, with larger values causing the distribution of the statistic to move from the non-standard DF distribution towards the standard normal. To examine whether the distribution of the $t_{\gamma}^{G L S}$ test exhibits similar behaviour, unit root processes $\left(y_{t}\right)$ in the above DGP are considered with varying forms of GARCH behaviour given by a range of combinations of the GARCH parameters $\left\{\phi_{1}, \phi_{2}\right\}$. The precise values selected are in part informed by estimated values observed in the empirical analysis of data of differing frequencies (see Drost and Nijman 1993; Engle and Patton 2001), where the volatility parameter $\phi_{1}$ is typically small relative to $\phi_{2}$ and the sum $\phi_{1}+\phi_{2}$ represents near integration. However, for completeness a full range of values is employed, including parameter combinations where $\phi_{1}$ is large relative to $\phi_{2}$. The exact values considered are: $\left\{\phi_{1}, \phi_{2}\right\}=\{0.05,0.90\}$, $\{0.15,0.80\},\{0.25,0.70\},\{0.35,0.60\},\{0.50,0.45\},\{0.70,0.25\},\{0.90,0.05\}$. Throughout, the intercept $\phi_{0}$ is given as $\phi_{0}=1-\phi_{1}-\phi_{2}$. While the initial value of $y_{t}$ is set to zero $\left(y_{0}=0\right)$, the initial value of the conditional variance is set equal to one $\left(h_{0}=1\right)$ following previous research (see, 
inter alia, Kim and Schmidt 1993; Seo 1999). The innovation series $\left\{v_{t}\right\}$ is generated using pseudo i.i.d. $\mathrm{N}(0,1)$ random numbers. Two sample sizes are considered for the experimental analysis, with $T=\{250,500\}$. For all experiments, an additional, initial 400 observations of the GARCH process $w_{t}$ are generated and discarded. For each of the experimental designs, the unit root hypothesis is examined over 25,000 replications via joint ML estimation of (8) and a $\operatorname{GARCH}(1,1)$ process using the Bernt-Hall-Hall-Hausman (BHHH) algorithm and the Bollerslev-Wooldridge covariance matrix estimator for data generated from (12)-(15). The testing equation of (8) is considered in two alternative forms as a result of differing options concerning the deterministic terms included, with $z_{t}=1$ or $z_{t}=(1, t)^{\prime}$. The $t_{\gamma}^{G L S}$ test is then calculated using (8)-(11) as explained in the previous section.

The finite-sample critical values for the $t_{\gamma}^{G L S}$ statistic are reported in Table One for the intercept and trend models. From inspection of Table One, it can be seen that the presence of GARCH results in more negative (greater in absolute value) critical values in comparison to those for the Elliott et al. (1996) test where GARCH is not present. However, as the sample size is increased the values become greater (less negative). This result was also found to hold by Cook (2007) for the DF-GARCH test. It can also be seen that the values of the GARCH parameters do have some impact upon the resulting critical values. In particular it can be seen that when either $\phi_{1}$ or $\phi_{2}$ move away from more moderate values, the observed critical values take more negative values.

\section{Table One about here}

\section{Monte Carlo analysis of power}

To examine the empirical power of the $t_{\gamma}^{G L S}$ test, the following data generation process (DGP) is employed:

$$
\begin{aligned}
y_{t} & =\rho y_{t-1}+w_{t} \quad t=1, \ldots, T \\
h_{t}^{2} & =\phi_{0}+\phi_{1} w_{t-1}^{2}+\phi_{2} h_{t-1}^{2} \\
w_{t} & =h_{t} v_{t} \\
v_{t} & \sim \mathrm{N}(0,1)
\end{aligned}
$$


The above DGP therefore closely mimics (12)-(15). Indeed, the previous Monte Carlo design is adopted regarding the number of replications employed, values of the GARCH parameters $\left\{\phi_{1}, \phi_{2}\right\}$, initial conditions and discarding of initial values of the GARCH process $\left\{w_{t}\right\}$. However, a departure from the previous DGP is concerns the introduction of the parameter $\rho$ to allow the analysis of test power. Via the imposition of $|\rho|<1$ it is ensured that $y_{t}$ does not possess a unit root, with the four near unity values of $\rho$ considered given as $\rho=\{0.980,0.985,0.990,0.995\}$. Additionally, in a further departure from the simulation of critical values, the power analysis conducted is performed for a single illustrative sample size of 500 observations $(T=500)$. As a result of the use of 7 different pairings of the GARCH parameters and four values of $\rho, 28$ different experimental designs are considered. For each of the experimental designs, empirical rejection frquencies at the $5 \%$ level of significance are calculated for the DF, DF-GARCH and DF(GLS)-GARCH tests. Each of these tests is considered with (i) an intercept and (ii) an intercept and linear trend included as deterministic terms. While the DF-GARCH and DF(GLS)-GARCH tests are denoted as $t_{\beta}$ and $\tau_{\gamma}^{G L S}$ under the use of both choices of deterministic terms, conventional notation is followed for the DF test with $\tau_{\mu}$ denoting the intercept model and $\tau_{\tau}$ denoting the trend model. The required critical values for the $\tau_{\mu}$ and $\tau_{\tau}$ tests are drawn from Fuller (1996), while the critical values for the $t_{\beta}$ and $\tau_{\gamma}^{G L S}$ tests are drawn from Cook (2007) and the analysis of the previous section respectively. In light of the dependence of the critical values for these tests upon the values of the GARCH parameters, critical values specific to the estimated value of the volatility parameter $\left(\phi_{1}\right)$ are employed.

The results of the power experiments are presented in Tables Two and Three. Considering the results for the intercept model in Table Two it is clear that the $t_{\gamma}^{G L S}$ test possesses far greater power than the $\tau_{\mu}$ and $t_{\beta}$ tests for all of the experimental designs considered. Interestingly, for designs with smaller values of the volatility parameter $\left(\phi_{1}\right)$, the power advantage of the $t_{\gamma}^{G L S}$ test is particularly noticeable. This feature is of interest as in empirical research estimated values of $\phi_{1}$ tend be similar to the smaller, rather than larger, values considered here. Considering the results for the trend model presented in Table Three, similar findings are observed with the $t_{\gamma}^{G L S}$ test again outperforming its rivals. In summary, the results of the simulation analysis show the $t_{\gamma}^{G L S}$ test to exhibit greater power than the $t_{\beta}$ test and the seminal DF test for both intercept and trend models in the presence of GARCH, particularly for more empirically realistic designs. 


\section{Tables Two and Three about here}

\section{An empirical analysis of UK output}

The above simulation analysis clearly depicts the power advantage of the $t_{\gamma}^{G L S}$ test relative to the $\mathrm{DF}$ and $t_{\beta}$ tests. To illustrate the empirical relevance of these findings, the tests are employed to examine the integrated nature of UK output. The data considered are quarterly observations on the natural logarithmic value of real GDP over the period 1955(1) to 2006(1). ${ }^{3}$ Given the clearly trending nature of the series, the rival tests are applied with an intercept and trend included as the relevant determinstic terms. The first test applied is the $\tau_{\tau}$ test. To determine the degree of augmentation of this test, the modified Akaike Information Criterion (MAIC) of Ng and Perron (2001) is employed following initial consideration of a maximum lag length $(p)$ given by $p=i n t$ $[12(T / 100)]^{0.25}$. Justification of this upper bound is provided by Hayashi (2000). The resulting calculated test statistic of -2.615 results in non-rejection of the unit root hypothesis when compared to the $5 \%$ critical value of -3.432 , or even the $10 \%$ critical value of -3.140 . However, consideration of the correlogram of squared residuals from the DF testing equation and application of an LM test of $\mathrm{ARCH}$, indicates the presence of conditional heteroskedasticity. As a consequence of this, the $t_{\beta}$ test is applied. Following the above approach to determine the degree of augmentation of the testing equation, application of the $t_{\beta}$ test using the BHHH algorithm and Bollerslev-Wooldridge robust standard errors results in a calculated test statistic of -3.075 . Given estimated, and highly significant, GARCH coefficients of $\left\{\phi_{1}, \phi_{2}\right\}=\{0.055,0.937\}$, the appropriate $5 \%$ critical value of -3.752 drawn from Cook (2007) shows that again the unit root null cannot be rejected. To explore the possibility that the higher powered $t_{\gamma}^{G L S}$ test may reject the null, it is applied using the BHHH algorithm and Bollerslev-Wooldridge covariance matrix estimator, with the same decision rule employed to determine the degree of augmentation of the testing equation. The resulting calculated test statistic is found to be -3.201 . Given estimated GARCH parameters of $\left\{\phi_{1}, \phi_{2}\right\}=$ $\{0.100,0.898\}$, the most appropriate critical values to draw from Table One are those for either $\left\{T, \phi_{1}, \phi_{2}\right\}=\{250,0.05,0.90\}$ or $\left\{T, \phi_{1}, \phi_{2}\right\}=\{250,0.10,0.85\}$. In either case it is clear that, in contrast to the results for the $\tau_{\tau}$ and $t_{\beta}$ tests, the null can be rejected at the $5 \%$ level of significance.

\footnotetext{
${ }^{3}$ The data were downloaded from the National Statistics Web site (http://www.statistics.gov.uk/). The series code is ABMI.
} 


\section{Conclusion}

In this paper, the literature on the examination of the unit root hypothesis in the presence of GARCH has been extended. Using local-to-unity detrending a new unit root test has been developed which involves joint maximum likelihood estimation of a unit root testing equation and GARCH(1,1) process. Using simulation analysis it was shown that this test possesses a clear power advantage relative to a previously proposed unit root test incorporating GARCH effects. To illustrate the empirical relevance of the simulation results, the order of integration of UK real output was examined. Following the failure of the seminal Dickey-Fuller test to reject the unit root hypothesis, it was noted that the residuals from the Dickey-Fuller testing equation exhibited conditional heteroskedasticity. In response to this find, the newly proposed test and previously proposed GARCH-based unit root tests were applied. While the latter test also failed to reject the null, the newly proposed test did reject the unit root hypothesis. The simulation and empirical analysis suggest the newly proposed test, which can be employed in both intercept and trend specifications, to be of value to practitioners interested in determining the order of integration of economic and financial time series processes.

\section{References}

[1] Anderson, T. and Bollerslev, T. (1998) 'Answering the skeptics: Yes, standard volatility models do provide accurate forecasts', International Economic Review, 39, 885-905.

[2] Bollerslev, T. (1986) 'Generalised autoregressive conditional heteroscedasticity', Journal of Econometrics, 31, 307-327.

[3] Bollerslev, T. and Wooldridge, J. (1992) 'Quasi-maximum likelihood estimation and inference in dynamic models with time-varying covariances', Econometric Reviews, 11, 143-172.

[4] Brooks, C. and Rew, A. (2002) 'Testing for a unit root in a process exhibiting a structural break in the presence of GARCH errors', Computational Economics, 20, 157-176.

\footnotetext{
${ }^{4}$ The Monte Carlo experimentation employed to derive this critical value follows the approach adopted above, with the error process generated in the same manner and an identical number of replications used.
} 
[5] Cook, S. (2002) 'Correcting size distortion of the Dickey-Fuller test via recursive mean adjustment', Statistics and Probability Letters, 60, 75-79.

[6] Cook, S. (2006a) 'The robustness of modified unit root tests in the presence of GARCH', Quantitative Finance, 359-363.

[7] Cook, S. (2006b) 'The impact of GARCH on asymmetric unit root tests', Physica A, 369, 745-752.

[8] Cook, S. (2006c) 'GARCH, heteroskedasticity consistent covariance matrix estimation and (non-)linear unit root testing', Applied Financial Economics Letters, 2, 217-222.

[9] Cook, S. (2007) 'Joint maximum likelihood estimation of unit root testing equations and GARCH processes: Some finite-sample issues', Mathematics and Computers in Simulation (forthcoming).

[10] Cook, S. and Manning, N. (2003) 'The power of asymmetric unit root tests under threshold and consistent-threshold estimation', Applied Economics, 35, 1543-1550.

[11] Dickey, D. and Fuller, W. (1979) 'Distribution of the estimators for autoregressive time series with a unit root', Journal of the American Statistical Association 74, 427-431.

[12] Drost, F. and Nijman, T. (1993) 'Temporal aggregation of GARCH processes', Econometrica, 61, 909-927.

[13] Elliott, G., Rothenberg, T. and Stock, J. (1996) 'Efficient tests for an autoregressive unit root', Econometrica 64, 813-836.

[14] Enders, W. (2001) 'Improved critical values for the Enders-Granger unit root test', Applied Economics Letters, 8, 257-261.

[15] Enders, W. and Granger, C. (1998) 'Unit root tests and asymmetric adjustment with an example using the term structure of interest rates', Journal of Business and Economic Statistics, 16, 304-311.

[16] Engle, R. (1982) 'Autoregressive conditional heteroscedasticity with estimates of the variance of UK inflation', Econometrica, 50, 987-1008.

[17] Engle, R. and Patton, A. (2001) 'What makes a good volatility model?', Quantitative Finance, 1, 237-245.

[18] Fuller, W. (1996) An Introduction Statistical Time Series (second edition), New Jersey: Wiley.

[19] Granger, C. and Hallman, J. (1991) 'Nonlinear transformations of integrated time series', Journal of Time Series Analysis, 12, 207-218.

[20] Haldrup, N. (1994) 'Heteroskedasticity in non-stationary time series: Some Monte Carlo evidence', Statistical Papers, 35, 287-307.

[21] Hayashi, F. (2000) Econometrics, Princeton: Princeton University Press.

[22] Kapetanios, G., Shin, Y. and Snell A. (2003) 'Testing for a unit root in the nonlinear STAR framework', Journal of Econometrics, 112, 359-379.

[23] Kim, K. and Schmidt, P. (1993) 'Unit root tests with conditional heteroskedasticity' Journal of Econometrics, 59, 287-300.

[24] Leybourne, S. (1995) 'Testing for unit roots using forward and reverse DF regressions', Oxford Bulletin of Economics and Statistics, 57, 559-571. 
[25] Leybourne, S., Mills, T. and Newbold, P. (1998) 'Spurious rejections by Dickey-Fuller tests in the presence of a break under the null', Journal of Econometrics, 87, 191-203.

[26] Leybourne, S. and Newbold, P. (2000) 'Behaviour of standard and symmetric Dickey-Fuller type tests when there is a break under the null hypothesis', The Econometrics Journal, 3, $1-15$.

[27] Maddala, G. and Kim, I.-M. (1999) Unit Roots, Cointegration, and Structural Change, Cambridge: Cambridge University Press.

[28] Mueller, U. and Elliott, G. (2003) 'Tests for unit roots and the initial condition', Econometrica, 71, 1269-86

[29] Ng, S. and Perron, P. (2001). 'Lag length selection and the construction of unit root tests with good size and power', Econometrica, 69, 1519-1554.

[30] Pantula, S., Gonzalez-Farias, G. and Fuller, W. (1994) 'A comparison of unit root test criteria', Journal of Business and Economic Statistics, 12, 449-459.

[31] Park, H. and Fuller, W. (1995) 'Alternative estimators and unit root tests for the autoregressive process', Journal of Time Series Analysis, 16, 415-429.

[32] Perron, P. (1989) 'The Great Crash, the oil price shock and the unit root hypothesis', Econometrica, 57, 1361-1401

[33] Perron, P. (1997) 'Further evidence on breaking trend functions in macroeconomic variables', Journal of Econometrics, 80, 355-385.

[34] Seo, B. (1999) 'Distribution theory for unit root tests with conditional heteroskedasticity', Journal of Econometrics, 91, 113-144.

[35] Shin, D. and So, B. (2001) 'Recursive mean adjustment for unit root tests', Journal of Time Series Analysis 22, 595-612.

[36] Taylor, S. (1986) Modelling Financial Time Series, New York: Wiley. 
Table One: Finite-sample critical values for the $t_{\gamma}^{G L S}$ test

The intercept model: $z_{t}=1$

\begin{tabular}{|c|c|c|c|c|c|c|}
\hline \multirow[b]{2}{*}{$\left(\phi_{1}, \phi_{2}\right)$} & \multicolumn{3}{|c|}{$T=250$} & \multicolumn{3}{|c|}{$T=500$} \\
\hline & $10 \%$ & $5 \%$ & $1 \%$ & $10 \%$ & $5 \%$ & $1 \%$ \\
\hline$(0.05,0.90)$ & -1.78 & -2.11 & -2.83 & -1.67 & -2.00 & -2.66 \\
\hline$(0.15,0.80)$ & -1.74 & -2.08 & -2.73 & -1.67 & -2.01 & -2.65 \\
\hline$(0.25,0.70)$ & -1.73 & -2.08 & -2.79 & -1.66 & -2.01 & -2.70 \\
\hline$(0.35,0.60)$ & -1.72 & -2.08 & -2.79 & -1.63 & -1.99 & -2.70 \\
\hline$(0.50,0.45)$ & -1.70 & -2.08 & -2.88 & -1.59 & -1.95 & -2.70 \\
\hline$(0.70,0.25)$ & -1.68 & -2.08 & -2.92 & -1.57 & -1.92 & -2.67 \\
\hline$(0.90,0.05)$ & -1.69 & -2.13 & -3.07 & -1.55 & -1.93 & -2.71 \\
\hline
\end{tabular}

The trend model: $z_{t}=(1, t)^{\prime}$

\begin{tabular}{|c|c|c|c|c|c|c|}
\hline \multirow[b]{2}{*}{$\left(\phi_{1}, \phi_{2}\right)$} & \multicolumn{3}{|c|}{$T=250$} & \multicolumn{3}{|c|}{$T=500$} \\
\hline & $10 \%$ & $5 \%$ & $1 \%$ & $10 \%$ & $5 \%$ & $1 \%$ \\
\hline$(0.05,0.90)$ & -2.73 & -3.08 & -3.84 & -2.61 & -2.90 & -3.49 \\
\hline$(0.15,0.80)$ & -2.62 & -2.93 & -3.53 & -2.56 & -2.86 & -3.46 \\
\hline$(0.25,0.70)$ & -2.60 & -2.95 & -3.57 & -2.53 & -2.85 & -3.46 \\
\hline$(0.35,0.60)$ & -2.60 & -2.96 & -3.69 & -2.49 & -2.86 & -3.54 \\
\hline$(0.50,0.45)$ & -2.58 & -3.01 & -3.92 & -2.47 & -2.86 & -3.68 \\
\hline$(0.70,0.25)$ & -2.56 & -3.04 & -4.21 & -2.47 & -2.91 & -3.96 \\
\hline$(0.90,0.05)$ & -2.58 & -3.11 & -4.60 & -2.49 & -2.97 & -4.23 \\
\hline
\end{tabular}

Notes: The figures in the above tables represent critical values for the $t_{\gamma}^{G L S}$ test at the $10 \%$, $5 \%$ and $1 \%$ levels of significance using (8)-(11) for data generated using the DGP of (12)-(15). Estimation was performed using the $\mathrm{BHHH}$ algorithm and the Bollerslev-Wooldridge covariance matrix estimator under alternative decisions concerning inclusion of deterministic terms. 
Table Two: Power analysis of unit root tests: The intercept model $\left(d_{t}=\alpha_{0}\right)$

\begin{tabular}{lllllll} 
& \multicolumn{5}{c}{$\left(\phi_{1}, \phi_{2}\right)$} \\
\cline { 2 - 5 }$(0.05,0.90)$ & $(0.15,0.80)$ & $(0.25,0.70)$ & $(0.35,0.60)$ & $(0.50,0.45)$ & $(0.70,0.25)$ & $(0.90,0.05)$ \\
\hline
\end{tabular}

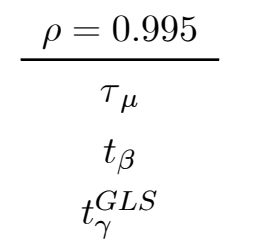

7.60

7.13

8.74

10.04

8.62

11.93

11.06

12.41

13.33

13.50

14.60

19.32

27.64

16.18

22.40

29.52

35.51

35.24

44.44

52.71

59.00

\begin{tabular}{c}
$\rho=0.990$ \\
\hline$\tau_{\mu}$ \\
$t_{\beta}$ \\
$t_{\gamma}^{G L S}$
\end{tabular}

12.13

13.64

15.47

17.07

18.88

19.78

19.85

10.71

14.92

23.30

33.27

45.63

58.08

66.91

31.79

41.73

53.96

63.16

73.33

80.49

85.63

$\rho=0.985$
${ }^{\tau_{\mu}}$
$t_{\beta}$
${ }_{t_{\gamma}}^{G L S}$

19.90

21.97

24.28

26.23

28.06

29.24

28.97

17.74

25.09

38.73

51.16

65.08

77.53

84.96

53.62

63.81

74.27

81.31

87.74

92.30

94.83

\begin{tabular}{c}
$\rho=0.980$ \\
\hline$\tau_{\mu}$ \\
$t_{\beta}$ \\
$t_{\gamma}^{G L S}$
\end{tabular}

31.37

33.48

35.96

37.73

38.85

40.02

39.61

27.73

38.42

54.35

67.36

80.23

89.14

93.72

73.00

80.20

86.72

90.75

94.38

96.84

97.96

Notes: The figures in the above table represent empirical rejection frequencies for the $\tau_{\mu}, t_{\beta}$ and $t_{\gamma}^{G L S}$ tests at the 5\% level of significance using the DGP of (16)-(19) over 25,000 replications. 
Table Three: Power analysis of unit root tests: The trend model $\left(d_{t}=\alpha_{0}+\alpha_{1} t\right)$

\begin{tabular}{lllllll} 
& \multicolumn{5}{c}{$\left(\phi_{1}, \phi_{2}\right)$} \\
\cline { 2 - 5 }$(0.05,0.90)$ & $(0.15,0.80)$ & $(0.25,0.70)$ & $(0.35,0.60)$ & $(0.50,0.45)$ & $(0.70,0.25)$ & $(0.90,0.05)$ \\
\hline
\end{tabular}

\begin{tabular}{|c|c|c|c|c|c|c|c|}
\hline$\rho=0.995$ & & & & & & & \\
\hline$\tau_{\tau}$ & 6.23 & 7.77 & 9.56 & 10.93 & 12.44 & 13.52 & 13.84 \\
\hline$t_{\beta}$ & 6.36 & 7.00 & 8.88 & 11.10 & 15.42 & 20.14 & 23.74 \\
\hline$t_{\gamma}^{G L S}$ & 7.17 & 8.28 & 10.38 & 12.95 & 16.38 & 19.38 & 23.09 \\
\hline \multicolumn{8}{|l|}{$\rho=0.990$} \\
\hline$\tau_{\tau}$ & 8.71 & 10.37 & 12.53 & 14.21 & 15.90 & 17.20 & 17.42 \\
\hline$t_{\beta}$ & 8.38 & 10.19 & 14.99 & 21.93 & 32.27 & 43.36 & 52.13 \\
\hline$t_{\gamma}^{G L S}$ & 12.16 & 14.89 & 21.04 & 27.49 & 35.32 & 42.92 & 50.26 \\
\hline \multicolumn{8}{|l|}{$\rho=0.985$} \\
\hline$\tau_{\tau}$ & 12.88 & 14.97 & 17.48 & 19.21 & 21.18 & 22.52 & 22.68 \\
\hline$t_{\beta}$ & 11.97 & 15.38 & 24.31 & 35.22 & 50.12 & 64.21 & 73.54 \\
\hline$t_{\gamma}^{G L S}$ & 20.22 & 25.49 & 35.18 & 44.10 & 54.62 & 63.32 & 71.07 \\
\hline \multicolumn{8}{|l|}{$\rho=0.980$} \\
\hline$\tau_{\tau}$ & 18.91 & 21.45 & 24.03 & 26.08 & 28.03 & 29.28 & 29.40 \\
\hline$t_{\beta}$ & 17.40 & 22.90 & 35.48 & 49.43 & 65.97 & 78.83 & 86.64 \\
\hline$t_{\gamma}^{G L S}$ & 31.83 & 39.16 & 50.16 & 59.50 & 69.42 & 77.67 & 84.21 \\
\hline
\end{tabular}

Notes: The figures in the above table represent empirical rejection frequencies for the $\tau_{\mu}, t_{\beta}$ and $t_{\gamma}^{G L S}$ tests at the $5 \%$ level of significance using the DGP of (16)-(19) over 25,000 replications. 